\title{
Oposición política al franquismo y exilio en Galicia: Estado de la cuestión
}

\author{
Fernando Prieto Valdés \\ Alberto Romasanta ARMEsto
}

\section{INTRODUCCIÓN}

El rápido triunto de las fuerzas sublevadas en el mes de Julio de 1936 supone para Galicia el paso, casi sin solución de continuidad, de la etapa republicana al período franquista. Dicho triunfo implica para muchos potenciales enemigos del nuevo régimen, el inicio de un largo exilio o, en su lugar, el comienzo de las primeras formas espontáneas de guerrilla.

El posterior desarrollo de los hechos a lo largo del periodo franquista llevaria consigo una alteración de la composición, perspectivas estrátegicas y tácticas mantenidas por la oposición gallega al franquismo, tanto en el exilio como en el interior. Entre los citados hechos habría que tener en cuenta un complejo conglomerado de factores que van desde la evolución de la coyuntura internacional hasta las propias transformaciones internas del franquismo, lógicas si tenemos en cuenta lo dilatado de su periodo de vigencia y que obligan a huir de cualquier intento simplificador a la hora de categorizarlo, pasando por las propias transformaciones de base de la sociedad gallega y, evidentemente, por la misma dialéctica interna de las fuerzas opositoras.

No de otro modo se puede seguir la evolución particular de la oposición gallega al franquismo, desde los intentos de reorganización de las fuerzas republicanas y las actividades guerrilleras de los años cuarenta 
hasta la emergencia de nuevas fuerzas, renovadas no sólo en lo ideológico sino también en sus bases sociales de apoyo, en los años sesenta, pasando por el diseño galleguista de una lucha centrada en las actividades culturales durante la década anterior. Es de destacar, asimismo, la especial tensión existente entre la oposición interna y el exilio, el cual, por otro lado, habría de perder protagonismo en los años finales del régimen, entre otras razones por la misma desaparición o envejecimiento físico de sus principales dirigentes.

En las páginas que siguen intentaremos describir el actual estado de la cuestión, bibliográfico y documental, de este importante aspecto de nuestro pasado reciente. El análisis de las fuentes documentales to hemos realizado clasificando las mismas en función de su carácter externo; por un lado fuentes escritas como las publicaciones de la oposición, la documentación inédita y la prensa periódica, y por otro las fuentes orales. En cuanto al repertorio bibliográfico, sin dejar de tener presente el diferente rigor y entidad de las obras que se citan, desde breves artículos a estudios de cáracter monográfico seriamente documentados, hemos preferido seguir para su clasificación un criterio temático. Tras una referencia de aquellas obras generales que pueden servir de introducción al tema, agrupamos el material bibliográfico disponible en dos grandes grupos, el de las obras referentes al exilio y el de aquellas otras, más numerosas, relacionadas con las actividades de la oposición interna. Esta última, a su vez, aparece subdividida bajo los epígrafes de "guerrilla", "oposición galleguista" y "otras oposiciones", apartado este último en el que las carencias son especialmente notables.

Podemos adelantar que los resultados de nuestro trabajo no son muy alentadores: la bibliografía existente es muy escasa y la mayoría de las fuentes son de difícil acceso y, por su propia naturaleza, dispersas, sobreabundantes y variadas. Esto es fácilmente justificable, en el caso de las fuentes, por la proximidad de los hechos estudiados. Dicha proximidad también explica la escasez bibliográfica, pero también es cierto que las orientaciones predominantes en la historiografia gallega contemporánea, y la existencia de unos ciertos prejuicios historiográficos a la hora de enfrentarse a nuestro pasado político reciente, no ayudan a paliar la actual carencia de estudios monográficos sobre la oposición y el exilio. Es por ello que no queremos poner fin a estas notas introductorias sin dejar de reivindicar la necesidad del estudio de esta parcela de nuestro pasado reciente y la superación de los prejuicios que aún hoy rodean a la "historia politica" y a la uhistoria del tiempo presente", sin cuyo conocimiento dificilmente se pueden comprender cabalmente algunas de las más decisivas determinaciones del momento presente de Galicia y del 
conjunto español. Y ello siendo conscientes de que la citada proximidad temporal del objeto de estudio exige del historiador tanto una especial metodología investigadora como una especial atención.

\section{FUENTES DOCUMENTALES}

El estudio de la oposición al franquismo en Galicia, en su doble vertiente de oposición interna y de exilio, exige la utilización de una gran variedad de fuentes que trasciende al mero documento inédito conservado en archivos, de acuerdo con la evolución de la disciplina histórica durante los últimos años ${ }^{1}$.

El carácter de estas fuentes, así como las posibilidades de acceso a las mismas, aparece profundamente condicionado, entre otros factores, por la relativa proximidad temporal del tema objeto de estudio. De este modo, si por un lado dicha proximidad facilita el empleo de fuentes "nuevas" (como pueden ser los testimonios orales o las grabaciones radiofónicas), por otro lado dificulta en cierta medida el acceso a determinados tipos de documentación, de un modo particular los documentos inéditos que, en buena parte, permanecen conservados en archivos personales, con lo cual su consulta depende en gran medida de la disposición de sus propietarios particulares.

En resumen, se pueden señalar como características definidoras de las fuentes disponibles para el estudio de la oposición al franquismo y del exilio en Galicia su pluralidad o variedad, su relativa «novedad", su dispersión (como se verá a continuación) y unas variables posibilidades de acceso a las mismas. A continuación se incluye una aproximación tipológica a dichas fuentes.

\footnotetext{
1 Tal y como lo manifiestan A. ALted y A. MATEOs para el conjunto de la oposición española: "La renovación de los estudios históricos a lo largo del presente siglo dentro de una dinámica pasado-presente, llevó al cuestionamiento de ese principio de validez única del documento escrito conservado en archivo, a la par que la utilización de nuevas fuentes ha provocado la revisión del concepto tradicional de archivo", en "Consideraciones en torno al carácter y significado de este Congreso", La oposición al régimen de Franco. Actas Congreso Internacional coordinado por TUSELL, J., ALTED A., y MATEOS, A., UNED, Madrid, 1990, vol. 1. pág. 28.
} 


\section{A) Publicaciones de la oposición.}

Al igual que en el resto de España, durante los años de vigencia del franquismo circuló por Galicia un elevado número de publicaciones clandestinas $^{2}$, con las cuales las organizaciones opositoras sacaban a la luz denuncias de la represión franquista y daban a conocer sus postulados a sus propios militantes y simpatizantes y a un más o menos amplio sector de la población gallega. Impresas unas clandestinamente en la propia Galicia, enviadas otras desde el exterior por los exilados, muchas de ellas tendrían una vida escasa, no pasando en ocasiones de uno o dos números.

Entre las publicaciones del interior ocupan un lugar preferente las de la guerrilla (E/ Guerrillero, Órgano del Ejército Guerrillero de Galicia, Senda Guerrillera, Vida Guerrillera, Combate, algunos periódicos murales de difusión meramente comarcal... ), las del Partido Comunista (Mundo Obrero, Organo del Comité de Galicia del Partido Comunista de España; Nuestra Bandera; A Voz do Pobo, Organo del P.C. de G. - cuya publicación se inicia en 1969, un año después de la "fundación gallega" del partido-... ) y las publicaciones vinculadas al nuevo nacionalismo de izquierdas surgido en la primera mitad de los años sesenta (como Adiante y Galicia Socialista, del PSG., o Terra e Tempo de la UPG, publicado desde 1965 - México para pasar luego a ser editado en Galicia-). Lógicamente, el número de publicaciones antifranquistas se multiplica en los últimos años del régimen, siendo particularmente activas en este campo las fuerzas de lo que se ha denominado "nueva izquierda" (comunistas ajenos al PC, trostkistas... ) y las organizaciones de masas y sindicatos que surgen o experimentan un gran desarrollo por estos años, destacamos entre otras:

- Servir al Pueblo del Movimiento Comunista de Galicia

- Canle, O Mallo y Terra e Tempo de la UPG

- Folga de las juventudes del PCG

- Lume del ERGA (Estudiantes Revolucionarios Galegos)

- Irmandiño del Frente Cultural Galego

2 Partiendo de los trabajos de B. Maiz M. Valcarcel y X. I. Taibo, recogidos en la bibliografía final, y de nuestra visita a algunas bibliotecas y archivos, hemos catalogado sobre cien publicaciones antifranquistas, entre las publicadas en el interior y las publicadas en el exilio. 
- Fouce de las Comisións Labregas.

También son muy numerosas las publicaciones del exilio, la mayoría procedentes de América y vinculadas a posiciones ideológicas próximas al nacionalismo (como son los casos de Adiante. Orgao das Mocedades Galeguistas (filial da I.G.), Consello de Galiza, Boletín informativo; Galicia Emigrante, A Nosa Terra -cuya publicación, interrumpida en el interior por el estallido de la guerra civil, se retoma en 1948 en América-, Vieiros - magníficamente impresa en México y transcendental como antecedente del nuevo nacionalismo de los años sesenta-... ). También en América verían la luz publicaciones de carácter unitario pero inspiradas por el Partido Comunista, como es el caso del Boletin Galego de Información y su continuadora Loita, promovidas en México por el comunista filo-nacionalista Luis Soto. Por último, como era de esperar dado el cambio de dirección de la corriente emigratoria gallega en los años sesenta, también en Europa se imprimirán publicaciones antifranquistas, como ocurre con la nueva Nova Galicia, sacada por el PC en Francia, o las publicaciones relacionadas con la Unión do Povo Galego que se introducen desde Ginebra.

En general, estas publicaciones, útiles no tanto por el mayor o menor grado de veracidad de sus informaciones sino en la medida en que ofrecen datos indispensables sobre los posicionamientos y estrategia de las fuerzas opositoras, se encuentran muy dispersas en un buen número de archivos personales, en archivos de esas mismas fuerzas (como los archivos del PCE en Madrid o de la UPG en Santiago, la Fundación Pablo Iglesias) y en diversas instituciones de índole cultural (la Biblioteca de la Universidad de Santiago, el Instituto Padre Sarmiento, también de Santiago, o la Fundación Penzol de Vigo, esta última con un amplio fondo relacionado sobre todo con el exilio americano y el nacionalismo en general.

\section{B) Documentación inédita}

Consideradas tradicionalmente como las fuentes principales de trabajo de los historiadores, devienen imprescindibles, aunque todavia de no fácil consulta, para conocer, entre otros aspectos, la vida interna y las expectativas de los circulos de opositores al régimen.

En esta categoria se incluirian desde documentación de tipo judicial y policial (conservada en los juzgados comarcales, los gobiernos civiles, el Servicio de Inteligencia de la Guardia Civil..., dicha documentación le sirvió a Bernardo Máiz para establecer un análisis sociológico de los 
integrantes de las guerrillas y de los detenidos por delitos de orden público entre 1965 y $1975^{3}$ ) hasta actas de reunión de organizaciones clandestinas o correspondencia particular de dirigentes y personas vinculadas con las actividades de oposición, tanto del interior como del exilio.

Al margen de la documentación policial y judicial, el resto de las fuentes englobadas en este apartado presenta también una gran dispersión. Junto a archivos de los partidos políticos (como el organizado, a principios de los setenta, por militantes de la UPG en Ginebra para huir de las pesquisas policiales en el interior, en el cual se conservan, entre otras cosas, la correspondencia mantenida por Celso Emilio Ferreiro con dicha organización en el período de su militancia en la misma) ${ }^{4}$, existen fundaciones como la "Fundación Castelao" que conserva la práctica totalidad del corpus epistolar del escritor y político de Rianxo, fondos de tipo privado (como los de los galleguistas Bieito Fernández y Manuel Beiras ${ }^{5}$-este último conserva, entre otros documentos de gran importancia, las actas de la reunión que mantuvo un representante del exilio con galleguistas del interior en 1958 en Santiago de Compostela- ${ }^{6}$ ) y documentación conservada en América, como es el caso de los archivos del "Consello de Galiza», conservados al parecer integros según noticias de I. Díaz Pardo.

\section{C) Fuentes orales}

Constituyen un tipo de fuentes que está alcanzando una gran utilización en los últimos años, a pesar de la tradicional imputación de "subjetividad" que se les hacia. Sin embargo, como expone L. Niethamer en su artículo "¿Para que sirve la Historia Oral?", esta es una técnica de investigación "adecuada para la exploración de determinados campos fragmentarios para los que no hay o a los que no es accesible otro tipo

${ }^{3}$ Maiz, B., Galicia en la ll República y bajo el franquismo. Vigo, Eds. Xerais, 1987, págs. 102 y 162).

4Algunas de estas cartas son reproducidas por C. X. Diaz MARTinez: "Militante da Unión do Povo Galego (UPG)", en VV.AA.: Celso Emilio Ferreiro. Común temos a patria. Vigo, A Nosa cultura 11, A Nosa Terra, 1989.

${ }^{5}$ De los que da cuenta NUNNEZ SEIXAS: "A supervivencia do nacionalismo galego na emigración americana, 1939-1960", en TuSELL, J., ALTED, A. y MATEOS, A. (coords.), op. cit., pág. 303.

${ }_{6}^{6}$ Un relato de esa misma reunión lo ofrece en sus memorias X. L. Franco Grande, uno de los participantes en ella: Os anos escuros, I. A resistencia cultural da xeración da noite (1954-1960). Vigo, Eds. Xerais, 1985, págs. 165 y 166. 
de documentos de transmisión"? ? En este sentido dichas fuentes son especialmente útiles en el campo que estamos analizando. De hecho, en Galicia, tales fuentes han sido usadas como complemento de la documentación escrita para estudios sobre organizaciones políticas del periodo republicano (el Partido Galeguista, la ORGA) y, en relación a la etapa franquista, han sido empleadas también por Bernardo Máiz, uno de los pioneros en su manejo para el período franquista. Por otro lado, recientemente se está creando un archivo de fuentes orales relacionadas con la represión y la resistencia al franquismo en la Facultad de Geografia e Historia de la Universidad de Santiago de Compostela ${ }^{8}$.

Sin ninguna duda, los testimonios orales pueden aportar muy útiles informaciones sobre la oposición y el exilio, ofrecidas por sus propios protagonistas directos. En este sentido, tanto valor tienen las entrevistas con dirigentes de organizaciones políticas como las que se puedan realizar con simples militantes, en la medida en que éstos pueden dar una imagen más cabal de las vivencias y las expectativas de todas aquellas personas que sufrieron la persecución (caso de los "fuxidos" de la guerra y de los primeros años cuarenta o de los detenidos en cualquier momento a lo largo de la vigencia del régimen de Franco) o el exilio, o tomaron parte activa en la resistencia interior militando en organizaciones clandestinas.

\section{D) Prensa periódica}

La consulta de las hemerotecas de los periódicos que se publicaban en Galicia durante estos años (La Voz de Galicia, El Correo Gallego, El Progreso, El Faro de Vigo, El ldeal Gallego, La Región, Diario de Pontevedra) puede aportar, sobre todo con posterioridad a la liberalización emprendida por Fraga Iribarne en 1966, noticias sobre la oposición en Galicia. Noticias que van desde referencias a detenciones de opositores a crónicas de los actos realizados por las fuerzas de oposición o comunicados emitidos por esas mismas fuerzas, especialmente en los últimos años de vigencia del régimen. pág. 5 .

7 Niethamer, L., “Para que sine la Historia Oral», Historia y fuente oral, núm. 2, 1989,

${ }^{8}$ El grupo que esta creando dicho archivo se denomina "Historga" y esta dirigido por Isaura Varela y Marc Wouters. 
Dicha información podría hallar su complemento en otras fuentes gráficas, como la fotografía, o en otros medios de comunicación, como es el caso de las grabaciones de emisiones radiofónicas.

\section{ESTADO DE LA CUESTIÓN BIBLIOGRÁFICO.}

\section{A) Bibliografía general}

Por lo general, la bibliografía disponible sobre la oposición y exilio gallegos durante el período franquista sigue siendo escasa y de muy desigual valor, incluyendo desde estudios realizados por historiadores profesionales a trabajos de corte periodístico. No existe aún un trabajo monográfico que presente una visión de conjunto del tema lo suficientemente documentado. Y ello a pesar del interés que por dicho tema surge, como era de esperar, en los años de la transición a la democracia.

En una primera aproximación pueden ser útiles los capitulos que, dentro de obras de carácter general, se le dedican al tema (BARREIRO FERNÁNDEZ $(1980,1981,1982,1988)$, CARBallo (1979) y VILLARES (1980, 1984) aunque, como es natural por obvias razones de espacio, no ofrezcan más que una visión en extremo sintética sobre los diferentes periodos de la oposición interior y sobre el papel jugado por el exilio, especialmente importante antes de 1950.

\section{B) La oposición interior}

\section{Aspectos generales}

En cuanto a la oposición interna, los mejores estudios para obtener una visión de conjunto son los de HEINE (1976) y MÁIz (1988). El primero de ellos, un artículo de apenas treinta páginas aparecido en los Cuadernos del Ruedo Ibérico, traza una visión sintética de la lucha opositora entre 1939 y 1975, contextualizándola en relación con las transformaciones de base operadas por la sociedad gallega a lo largo de este período. Presta una especial atención al nuevo nacionalismo surgido en la primera mitad de los años sesenta (PSG y, sobre todo, UPG) de resultas de la 
crisis del "Consello da Mocedade", utilizando fundamentalmente publicaciones diversas de las mismas organizaciones opositoras.

Por su parte Málz, B., autor de una tesis no publicada con el título de "1936-1964. A esquerda militante en Galicia» (leída en la Universidad de Zaragoza en 1984), es uno de los investigadores que más han trabajado sobre el tema de la oposición al franquismo en Galicia. Dedica un amplio capítulo de su libro Galicia na // República e baixo o franquismo a reconstruir las actividades de la lucha anti-franquista, evidenciando un amplio manejo de fuentes (judiciales, orales, publicaciones opositoras... ), aunque la obra peque a veces de la utilización de una terminologia equívoca ("oposición activa" versus "oposición cultural» para el período de 1936 a 1954) o de una cierta actitud "militante" que implica un escaso distanciamiento con respecto a la materia objeto de estudio. De todos modos, tanto esta obra como la comentada de $\mathrm{H}$. Heine superan con mucho a trabajos como el de Costa Clavell (1977), en exceso anecdótico y un tanto sensacionalista, obra de un historiador no profesional que da la impresión de estar elaborada un tanto precipitadamente, al amparo de la demanda de este tipo de libros suscitada por la muerte de Franco. Algún dato interesante aporta la obra del portugués LoY RoLLIM (1977) que dedica 15 páginas del capítulo "As nacionalidades" a Galicia en su obra $A$ oposiçao em Espanha. Un breve estudio del sistema de partidos antes de las elecciones de 1977, dando especial importancia a los grupos nacionalistas lo realizan, PRADA (1977), CORES TRASMONTE (1981) y BUSE (1984) en obras de cáracter general.

Igualmente interesantes para el conocimiento del tema son los dos números especiales de $A$ Nosa Terra, publicados en 1987 y 1988 y dedicados el primero (coordinado por G. Luca de Tena, X. Carballa y F. Carballo) al período 1936-1953 y el segundo (bajo la coordinación de X. Carballa, A. L. Carreira y L. Obelleiro) a la etapa que transcurre entre esa última fecha y la muerte de Franco. En ellos se hace referencia a las actividades de la oposición (artículos de B. Máiz y F. Carballo, fundamentalmente) y a los conflictos y movimientos sociales del momento, prestándole una atención especial a los movimientos de orientación nacionalista más recientes.

Otra obra que sigue siendo útil es la firmada conjuntamente por RIVAS, M., y TAlBO, X. I. (1977), en la que se hacía un repaso a las formaciones políticas existentes en el momento, desde la extrema izquierda a la extrema derecha, agrupándolas en dos grandes apartados («Os grupos políticos exclusivamente galegos" y "Os partidos operantes a nivel do Estado Español») y con interesantes referencias a sus orígenes y pasado. No ocurre exactamente lo mismo con un libro publicado por FERNÁN- 
DEZ, C., en 1985, el cual —pese a su prometedor título: Franquismo y transición política en Galicia- consiste básicamente en una mera recopilación de pequeños fragmentos de temática diversa (desde valoraciones globales a simples anécdotas). Lo más interesante de la obra quizás resulte ser la inclusión de documentos de cierto valor, como el "Manifiesto de la Alianza Nacional de Fuerzas Democráticas", difundido en Galicia en septiembre de 1945, o cartas personales de destacados personajes de la oposición (Castelao, Salvador de Madariaga, Sánchez Albornoz, los guerrilleros M. Ponte y Gayoso... ).

Por último, dentro de las obras integradas en el repertorio bibliográfico que más abajo se incluye bajo el epigrafe de "aspectos generales", son de destacar también:

- Estudios dedicados a analizar la situación de la lengua gallega (aspecto capital, el de su reivindicación, de los programas de las organizaciones nacionalistas, asumido con mayor o menor coherencia en los últimos años del franquismo por la práctica totalidad de las fuerzas opositoras), como los de ALONSO MONTERO (1973; 1974), militante o cuando menos próximo ideológicamente por aquellos años al Partido Comunista de Galicia, y Rodriguez, F. (1976), destacado militante de la UPG, cuya obra constituye un breve pero intenso estudio destinado, entre otras cosas, a combatir lo que denomina "tesis bilingüistas" (entre las que incluye la de la "muerte de la lengua" de Alonso Montero) desde una perspectiva de nacionalismo radical.

- Trabajos, de gran utilidad para los investigadores, sobre la prensa clandestina durante el franquismo, como los publicados por TAIBO (1977) VALCÁRCEL (1982) O MÁlz (1989).

- $Y$, finalmente, algunos relatos novelados referidos a los momentos iniciales de la represión desatada por el nuevo poder triunfante en Galicia ya en 1936 y a las primeras formas de resistencia. Entre ellos ocupan un lugar especial los de VALENZUELA, R. (1976; 1980), militante del Partido Galleguista durante la República e incorporado al P. Comunista en el periodo franquista.

\section{La guerrilla}

Uno de los episodios más espectaculares de la lucha interior contra el franquismo que comienza a ser bastante bien conocido es el de la guerrilla, forma de resistencia dominante durante los años cuarenta, desde sus origenes en los "fuxidos do monte" hasta la posterior conversión de sus supervivientes en una suerte de bandoleros rurales que continua- 
ban en la lucha armada por imperativos de mera supervivencia, pasando por su etapa de mayor auge coincidiendo con los años finales de la II Guerra Mundial y los comienzos de la segunda mitad de los cuarenta, años en los que se acentúa el control sobre ella ejercido por el Partido Comunista.

La obra hasta el momento "canónica» sobre este tema es la publicada por HEINE en 1980, la cual cuenta con el mérito añadido de contener interesantes referencias al conjunto de la oposición gallega en el período de vigencia de la lucha guerrillera. Este estudio se completa con los trabajos de Reigosa, Fuxidos de sona (1989) y Fernández, Carlos (1988), con algún capítulo publicado anteriormente en la Gran Encilopedia Gallega, realizado desde una óptica periodística, de MÁlz (las voces "Guerrilla" y "Ponte" en la GEG, la colaboración en el especial de $A$ Nosa Terra dedicado al período 1936-1953 y un apartado en su libro Galicia na // República e baixo o franquismo) y de LOPEZ RICO, J., en la $G E G$. Existen otros trabajos que, aunque estudian el tema de la guerrilla a nivel estatal, también contienen referencias a Galicia, por ejemplo el documentado trabajo de VILLANUEVA (1977) o las obras de AGUADO SANCHEZ (1975 y 1976), que representan el análisis oficioso del hecho guerrillero, muy completo en datos y documentación, al ser su autor un jefe de la Guardia Civil.

\section{La oposición galleguista}

También comienza a ser bastante bien conocida, al menos en sus lineas generales, la historia del nacionalismo gallego bajo el franquismo. Representado mayoritariamente antes de la guerra por el Partido Galeguista (del cual se separarían algunos elementos derechistas antes del estallido del conflicto civil), la represión de los primeros años que siguieron a la victoria del franquismo se abatiría sobre sus filas al igual que sobre el resto de las organizaciones fieles a la República. Hacia finales de los años cuarenta, desaparecidas las esperanzas de una reimplantación de la República como consecuencia de la victoria aliada en la Guerra Mundial y luego de unos años de contactos con otras fuerzas opositoras, en un contexto de gran debilitamiento orgánico (con muchos dirigentes y militantes desaparecidos, otros, como Castelao o Suárez Picallo, en el exilio), el galleguismo optaria por una lucha preferentemente cultural de recuperación de las señas de identidad del pueblo gallego (fundación de la editorial Galaxia, publicación de las revistas Grial y Revista Económica de Galicia...), abandonando las actividades políticas en franca oposición a las tesis defendidas por los galleguistas del exilio. De ahí que, 
en la primera mitad de los años sesenta, en una suerte de cruptura generacional", nuevas organizaciones ahora claramente de izquierdas vendrian a ocupar el hueco dejado por la liquidación del histórico PG.

En 1963 la aparición del Consello da Mocedade supone el reinicio de la oposición en Galicia y la reacción a la tendencia cultural de los hombres de Galaxia. Este nuevo grupo aglutinará corrientes nacionalistas políticas muy diferentes que pronto habrian de cuajar en partidos políticos como la UPG, el PSG y otros partidos de orientación socialdemócrata o demócrata-cristiana que, junto al Partido Comunista de Galicia constituido en París en 1968, protagonizarán la oposición al franquismo durante los años sesenta y setenta. A partir de 1975 estos grupos organizarán el Consello de Forzas Políticas Galegas en el que se agrupaban los partidos que se consideraban "genuinamente galleguistas": Partido Socialista Galego, Unión do Pobo Galego y Partido Galego Social-Democráta. En abril el Consello aumentó con la incorporación del Movimiento Comunista de Galicia y el Partido Carlista de Galicia.

De todos modos, se siguen echando en falta monografías rigurosas sobre el tema. Al margen de las obras de tipo general ya citadas, se puede obtener una imagen global del nacionalismo de postguerra en $\mathrm{V}_{1}$ LAS NOGUEIRA (1977), quien publica un artículo en 1977 en la revista Teima de reducidas dimensiones dada la naturaleza del medio en el cual aparece. Enmarcándolo dentro de la evolución histórica general del galleguismo, desde sus origenes provincialistas en la centuria pasada, revisten interés también la voz "Galeguismo" de BARREIRO, X. R., aparecida en la GEG, y un articulo de MAIZ, R., "El nacionalismo gallego: apuntes para la historia de una hegemonia imposible» incluido en 1986 en un libro colectivo sobre la cuestión nacional en España. Igualmente, un apartado del estudio dedicado por CASTRO, X. (1985) al galleguismo republicano ofrece información sobre el galleguismo del interior y del exilio en los primeros años del período franquista.

En el capítulo de las memorias, siempre de tanto interés para reconstruir las actividades opositoras, existe todavía una gran escasez de publicaciones. Son de destacar el primer tomo de las memorias de Franco GrandE, X. I. (1985), dedicado a los años que van desde 1954 a 1960 y que constituye un documento de primera mano sobre las actividades $y$ los componentes ideológicos (liberalismo, federalismo europeísta, anti-comunismo...) del galleguismo culturalista liderado por el recientemente fallecido Ramón Piñeiro. También se cuenta con unas memorias de Soto, Luis (1983), histórico militante comunista del ala galleguista durante la República y fundador posteriormente de la UPG, las cuales abarcan desde 
el periodo bélico hasta los primeros años ochenta y contienen informaciones sobre sus relaciones con Castelao, la experiencia del exilio mexicano o las vicisitudes del nuevo nacionalismo de izquierdas, entreveradas todas ellas con frecuentes disquisiciones de carácter doctrinario.

En un plano semejante a las memorias se sitúan algunos libros de entrevistas (»conversas"). El primero en publicarse fue el de FreiXanes (1976), con conversaciones entre las que se incluian alguna con destacados personajes de la oposición al franquismo como R. Piñeiro, X. M. Beiras o X. L. Méndez Ferrín. Con posterioridad, ya en 1989, aparecieron las de Salgado y Casado con Méndez Ferrin, y las de Fernán Vello y PILLADO MAYOR con Beiras, destacados dirigentes ambos del nacionalismo surgido en los años sesenta. También está a punto de aparecer la de Linares GIRAut (SE) con Pousa Antelo, histórico dirigente del PG. Los tres libros son fundamentales para conocer, con testimonios de primera mano, la evolución del nacionalismo gallego desde la época de predominio de la línea "culturalista» (años cincuenta) hasta el posterior camino seguido por las organizaciones (PSG y UPG) aparecidas, parcialmente al menos, como contestación a esta línea en 1963 y 1964, respectivamente.

La visión se completa con las desiguales voces dedicadas al "Partido Socialista Galego" y a la "Unión do Povo Galego" en la GEG, de mucha mayor entidad la primera, escrita por destacados dirigentes en su momento de dicho partido (Mario Orjales, Manuel Caamaño y X. M. Beiras), y algunos artículos incluidos en el especial de $A$ Nosa Terra dedicado al período 1953-1975, en especial los referentes a las organizaciones de masas potenciadas por la UPG en los últimos años del franquismo. Por otra parte, obras como las de Beiras $(1972 ; 1984)$ o la de RODRIGUEZ y LOPEZ SUEVOS (1978) sirven para conocer los análisis y postulados del nacionalismo de izquierdas desde dentro.

\section{Las otras oposiciones}

Mucho peor conocidas son las otras oposiciones, de ellas la bibliografía hasta ahora existente es muy escasa. Sin embargo, su evolución merecería el estudio de los especialistas. Cuando estalla la guerra civil la oposición es casi totalmente desarticulada y solo dos grupos logran reorganizarse:

- EI PCE, con sus principales dirigentes gallegos, Lister y Santiago Álvarez, en el exilio, comenzara a reorganizarse en el interior a partir de 1942 en las cárceles, su principal actividad será la organización de las guerrillas. 
- La CNT se reorganiza en 1942 y es uno de los grupos más activos de la oposición hasta que en 1950 fueron detenidos sus principales dirigentes.

Mientras tanto las numerosas personalidades gallegas encuadradas en partidos republicanos se integran en la oposición con sus respectivos grupos. Tan solo hay un intento de formar un bloque republicano gallego en el exilio en 1944, cuando, bajo la presidencia de Portela Valladares, se organiza en Toulouse el Bloque Republicano Nacional Galego con políticos como Casares Quiroga de IR o Enrique Lister del PCE,... y ningún miembro del Partido Galeguista en el consejo directivo.

Durante los años sesenta y setenta la principal organización opositora sera el PCE parcialmente "galleguizada" desde 1968 (conversión de la organización gallega del partido en Partido Comunista de Galicia), galleguización que contaba con antecedentes en algunos dirigentes del período republicano (como Luis Soto o Benigno Álvarez), por otro lado la protesta estudiantil en Santiago y el movimiento obrero en Ferrol y Vigo se convertirán en los principales focos de oposición al franquismo.

En los años setenta los partidos de oposición no nacionalistas siguieron un camino paralelo a la oposición de todo el Estado. En marzo de 1975 se había constituido en Viana do Castelo la Xunta Democrática de Galicia formada, sin la participación de los nacionalistas, por el Partido Comunista de Galicia, el Partido Socialista Popular Gallego, el Partido do Traballo de España, Comisiones Obreras, y personalidades independientes como Víctor Moro, Paz Andrade, Álvarez Gándara y Garcia Agudín, que representaría a la Xunta en la "cumbre" de la oposición celebrada en París a principios de 1976. Sin embargo la Xunta siempre buscó la unidad de todas las fuerzas de la oposición, pero sus esfuerzos resultaron inútiles, el 13 de julio del 76 se constituye la Táboa Democrática sin la participación de los nacionalistas, tuvo que conformarse con aglutinar a los miembros de la Xunta, excepto los independientes, y a la Federación Socialista Galega de PSOE, a la Organización Revolucionaria de Trabajadores y a los sindicatos Unión Sindical Obrera, Información Obreira y Unión General de Trabajadores. Ni siquiera logró atraer a la democracia cristiana del Partido Popular Galego ni a ningún grupo de la derecha democrática. Aunque con ellos designó al representante de Galicia en la comisión negociadora de la oposición a nivel estatal: Valentín Paz Andrade.

De todas estas actividades apenas tenemos estudios, ya que aparte de los trabajos ya comentados dedicados a la guerrilla, cuyo principal apoyo e impulsor fue el PC, y de las obras de carácter general a que 
también ya se ha hecho referencia (en especial la de Bernardo Máiz -1988- y la de Rivas y Taibo - 1977- para los años setenta), se carece casi por completo de estudios específicos sobre las formas opositoras no nacionalistas, siendo únicamente de destacar algunas voces en la GEG y las memorias de PORTELA VALLADARES (las cuales abarcan un arco cronológico que va desde 1916 a 1950) y las del dirigente comunista Álvarez, S., en varios volúmenes que se inician en el período de su militancia en el PC de la República.

\section{C) El exilio}

Gran importancia en la historia de la Galicia contemporánea tiene el exilio republicano, preferentemente dirigido a América, lo cual no es de extrañar si tenemos en cuenta el elevado número de gallegos emigrados en aquellas tierras (a comienzos de los años cuarenta Buenos Aires era la "ciudad gallega" más grande del mundo, por el número de gallegos residentes en ella), emigrantes a los que se vendrían a añadir los exilados por motivos políticos al finalizar la guerra. Teniendo como principales núcleos Argentina, México, Uruguay o Cuba, estos exilados se esforzarán por movilizar al entramado tejido de las sociedades gallegas en América a favor de la causa republicana. Así, se crearían instituciones culturales, como el "Padroado da Cultura Galega de México" que unos años más adelante publicaría la histórica revista Vieiros, u organizaciones de tipo político como la "Irmandade Galega", especie de continuadora del PG en tierras americanas, o el "Consello de Galiza" (formado en 1944 por algunos diputados gallegos -Castelao, Suárez Picallo, Elpidio Villaverde y Alonso Ríos, a los que más tarde se añadiria A. Somoza- como fideicomisario de la voluntad democrática del pueblo gallego expresada en el plebiscito del Estatuto de Autonomía).

La parte mejor conocida del exilio gallego (en gran parte gracias a la labor de recuperación de esta parcela de la memoria histórica emprendida por Isaac Díaz Pardo al frente de sus Edicións do Castro) la constituye el exilio galleguista. Básicos para su conocimiento son trabajos como los de NúNEEZ SeIXAS (1990), en el que se traza un breve pero bastante completo panorama de la evolución del exilio gallego en América hasta 1960, justamente en el momento en que dicho exilio comienza a perder peso político por el desarrollo de una oposición interior y el natural envejecimiento de sus protagonistas. En realidad, ya desde la muerte de Castelao, acaecida en 1950, el exilio americano habia sufrido un duro golpe y tan solo un año después fallecía Alfredo Somoza, primo 
del anterior militante de Izquierda Republicana y de quien contamos con una documentada biografía debida a SIXIREI, C. (1987)), o el de BIEITO CUPEIRO (1989), centrado en el exilio bonaerense y en el que se presta una atención especial a las diferencias internas entre los exilados y a las vicisitudes del "Consello de Galiza", ofreciendo una interpretación muy distinta de la que nos da D IAZ PARDO (1987) sobre su reorganización/ ampliación de 1960, autor este último que considera dicha reorganización como una alteración sustancial de su función de "fideicomisario". Igualmente, aunque abarcan un período cronológico más vasto que el que corresponde al exilio, siguen siendo fundamentales las obras de VILANOVA RODRíguez y ZUBILlaga B ARRERA (1966), publicadas en América y dedicadas a la emigración argentina y uruguaya, respectivamente.

Como es natural, dada su dimensión política y su capacidad para actuar de aglutinante de los diferentes grupos de exiliados, la figura de Castelao ocupa un lugar preferente dentro de la bibliografía existente sobre el exilio. Dos biografias, la de NuÑEz BUA, escrita el mismo año de la muerte de Castelao y publicada en Galicia en forma de libro por Edicións do Castro, y la de PAZ ANDRADE (1982), compañero de partido del político e intelectual rianxeiro durante los años de la República, hasta ahora la más amplia y documentada, dedican parte de sus páginas a narrar la actividad de Castelao en el exilio. A ellas viene a sumarse la monografía de NEIRA VILAS (1983) sobre el paso de Castelao por la isla de Cuba y la reciente publicación, por $A$ Nosa Terra, de una selección del corpus epistolar (incluyendo cartas a J. A. Aguirre, Martínez Barrio, Portela Valladares...) de esta figura transcendental en la historia reciente de Galicia.

Por último, dejar constancia de la existencia de dos memorias, la de Portela Valladares (1988), con un último capítulo dedicado a su experiencia en el exilio, y la de Soto, Luis (1983), con abundantes referencias a su exilio mejicano.

\section{REFERENCIAS BIBLIOGRÁFICAS}

\section{A) Obras generales}

Barreiro Fernández, X. R., "Galicia no século XX", en Galicia Eterna. Historia. Barcelona, Planeta, 1980.

- Historia de Galicia. IV: Edade Contemporánea. Vigo, Galaxia, 1981. 
- Historia de Galicia, ts. XV, XVI y XVII. La Coruña, Ganma, 1982.

- "Galicia en los siglos XIX y XX», en Enciclopedia Temática de Galicia. Barcelona, Nauta, 1988.

Carballo, F., “Apéndice. A Edade Contemporánea desde 1939 até 1979", en BARReIRo, X. R. et al., Historia de Galicia. Pontedeume, Frente Cultural da AN-PG, 1979.

Villares, R., «Idade Contemporánea», en Bermejo, X. C. et al., Historia de Galiza. Madrid, Alhambra, 1980.

- A Historia. Vigo, Galaxia 1984.

B) La oposición interior:

1. Aspectos generales:

Alonso MONTERo, X., Informe dramático sobre la lengua gallega. Madrid, Akal, 1973.

- Encuesta mundial sobre la lengua y cultura gallegas. Madrid, Akal, 1974.

- "Literatura galega de 1936 a 1945. Algúns aspectos da represión lingüistica", en A nosa historia 2. Os anos despois (1936-1953). Vigo, A Nosa Terra, 1987.

BuSE, M., La nueva democracia española. Madrid, Unión Editorial, 1984, págs. 367-373.

Carballa, $X$., "Notas sobre a avaliación da represión e a morte violenta despois de 1936", en A nosa historia 2. Os anos despois...

- "Castrelo, historia dun val afogado", en $A$ nosa historia 5. Galiza: 1953-1975. Vigo, A Nosa Terra, 1988.

ChaO, R., Después de Franco, España. Madrid, Felmar, 1976.

Cores TRAsmonte, B., "Modelos organizativos, participación y conciencia politica en Galicia", Documentación Social, núm. 45, (Octubre-Diciembre 1981)

Costa Clavell, X., Las dos caras de Galicia bajo el franquismo. Madrid, Ed. Cambio 16, 1977.

Diaz Fernandez, X., Os que non morreron. Sada-A Coruña, Eds. do Castro, 1982.

Duran, J. A. (coord.), Galicia, realidade económica e conflicto social. Bilbao, Servicio de Publicaciones del Banco de, 1978, s. e.

FERnÁNDEZ, C., Franquismo y transición política en Galicia. Sada-A Coruña, Eds. do Castro, 1985. 
Fernandez, S. y Brocos, M., Galicia Hoy. París, Ruedo Ibérico, 1966.

HEINE, H., "La evolución política de Galicia (1939-1975)", Cuadernos del Ruedo Ibérico, núm. 51-53 (mayo-octubre 1976).

Prada Fernández, J. L., “El sistema de partidos políticos en Galicia, una aproximación descriptiva” en VEGA, P., Teoría y práctica de los partidos políticos. Madrid, Edicusa, 1977.

RoLIN, L., A oposiçao em Espana. Lisboa, Ed. Gleba Lda., 1977.

MÁlZ, B., "A oposición política até 1975", en A nosa historia 5. Galiza: 1953-1975...

- Galicia na II República e baixo o franquismo (1930-1976). Vigo, Eds. Xerais, 1988.

MÁlz VÁzQUEZ, B., Memoria-catálogo das publicacións galegas anti-franquistas. Sada-A Coruña, Eds. do Castro, 1989.

Málz SUÁREZ, Ramón, s.v. "Franquismo", Gran Encilopedia Gallega, t. 14.

Méndez DomeneCH, X. M, “O 68 galego», en A nosa historia 5. Galiza: 1953-1975...

OJEA, A., “Vigo, 1972», en A nosa historia 5. Galiza: 1953-1975...

RIVAS, X. M., y TAIBO, X. I., Os partidos políticos na Galiza. A Coruña, Eds. do Rueiro, 1977.

Rodríguez, F., Conflicto lingüístico e ideoloxía en Galicia. Monforte de Lemos, Xistral, 1976.

SANFIZ, E., "O grao de area do Ferrol contra a Dictadura", en $A$ nosa historia 5. Galiza: 1953-1975...

TAlBO, X. I., «Prensa política en Galicia», Teima, núm. 19 (21-28-4-1977).

VÁlCARCEL, M., “A prensa clandestina na Galiza do franquismo», A Nosa Terra, núm. 189-190-191 (maio-xuño 1982).

ValenzUela, R., Non agardei por ninguén. Madrid, Akal, 1976.

- Era tempo de apandar. Madrid, Akal, 1980.

VV. AA., A nosa historia 2. Os anos despois (1936-1953). Vigo, A Nosa Terra, 1987.

VV. AA., A nosa historia 5. Galiza: 1953-1975. Vigo, A Nosa Terra, 1988.

2. La Guerrilla:

Aguado SÁnchez, El maquis en España. Madrid, San Martin, 1975.

- El maquis en sus documentos. Madrid, San Martin, 1976.

Fernández, C., La guerra civil en Galicia. La Coruña, La Voz de Galicia, 1988.

Freixanes, V. F., Memorias dun fuxido. O Fresco. Vigo, Eds. Xerais, 1980. 
HeINE, H., A guerrilla antifranquista en Galicia. Vigo, Eds. Xerais, 1980. LÓPEz Rico, Juan, s.v. "Gafas", G.E.G., t. 14.

- s.v. "Piloto", G.E.G., t. 24.

MAIZ, B., "A resistencia armada ao franquismo", en A nosa historia 2. Os anos despois (1936-1953) ....

MÁlz VÁZQUEZ, Bernardo, s.v. "Guerrilla», G.E.G., t. 17.

- s.v. «Ponte Pedreira, Manuel», G.E.G., 25.

Reigosa, Gonzalez, Carlos, Fuxidos de sona. Vigo, Eds. Xerais, 1989.

- s.v. "Foucellas", G.E.G., t. 14.

- S.v. "Gayoso", G.E.G., t. 15.

VILLANueVA, M., Les guerrillas en Léon-Galicia de 1937 á 1949. Université de Paris-Sorbonne,1977.

3. Oposición galleguista:

Barreiro Fernández, José Ramón, s.v. "Galeguismo», G.E.G., t. 15.

BeIRAS, X. M., O atraso económico de Galicia. Vigo, Galaxia, 1972.

- Por unha Galicia liberada. Ensaios en economía e política. Vigo, Eds. Xerais, 1984.

Caamaño Suárez, Manuel; Orjales Pita, Mario; Bieras, Xóse Manuel, S.v. "Partido Socialista Galego", G.E.G., t. 24.

Carballo, F., «Pensamento político na década dos 60 ", en $A$ nosa historia 5. Galiza: 1953-1975...

CASTRo, X., O galeguismo na encrucillada republicana, Deputación Provincial de Ourense, Ourense, 1985, t. 2.

fernan Vello, M. A. y Pillado Mayor, F., A nación incesante. Conversas con X.M. Beiras. Barcelona, Sotelo Blanco, 1989.

FERnÁNDEZ, C., La persecución de Castelao durante el franquismo. SadaA Coruña, Eds. do Castro, 1986.

Freixanes, V. F., Unha ducia de galegos. Vigo, Galaxia, 1976.

Franco Grande, X. L., Os anos escuros (1954-1960). I: A resistencia cultural da xeración "da noite». Vigo, Eds. Xerais, 1985.

Liñares Giraut, A., Conversas con Avelino Pousa Antelo, s.e.

Lopez Carreira, A., "ERGA, o lume que prendeu», A nosa historia 5. Galiza: 1953-1975...

MÁZ, R., "El nacionalismo gallego: apuntes para la historia de una hegemonía imposible", en HERNÁNDEZ, F. y MERCADE, F., Estructuras sociales y cuestión nacional en España. Barcelona, Ariel, 1986.

MERA, M., «Actividade sindical e nacionalismo", en $A$ nosa historia 5. Galiza: 1953-1975... 
MuÑIz, R., “Historia das Comisións Labregas", en A Nosa historia 5. Galiza: 1953-1975...

Rodríguez, F. y LOPEZ Suevos, R., Problemática nacional e colonialismo. O caso galego. Monforte de Lemos, Xistral, 1978.

Salgado, X. M. y CaSAdo, X. M., Xosé Luis Méndez Ferrín. Barcelona, Sotelo Blanco, 1989.

Soto, L., Castelao, a U.P.G. e outras memorias. Vigo, Eds. Xerais, 1983. s.v. "Unión do Povo Galego", G.E.G., t. 29.

VILAS NOGUEIRA, $X$., "O nacionalismo galego baixo o franquismo". Teima, núm. 32 (22-28-7-1977).

4. Otras oposiciones:

Álvarez, S., Unha alternativa democrática para Galicia. París, Nova Galicia, 1976.

- "Galicia y su problema nacional», en VV. AA., Asalto al centralismo. Barcelona, Avance, 1976.

- Ensaio encol do problema nacional galego. Madrid, Akal, 1976.

- ¿Qué es el P.C.G.? Madrid, Akal, 1977.

- Galicia, nacionalidad histórica. Madrid, Ayuso, 1980.

- Memorias, 3 vols., Sada-A Coruña, Eds. do Castro, 1985...

- Castelao y nosotros los comunistas. Sada-A Coruña. Eds. do Castro, 1984.

Gónzalez Probados, Manuel; Guerreiro Carrejra, Anxel, s.v. «Partido Comunista de Galicia», G.E.G., t. 24.

s.v. "Movemento Comunista de Galicia», G.E.G., t. 22.

Palomares Ibáñez, Jesús Maria; Durán, José Antonio; Gonzalez ProBADOS, Manuel, s.v. “Socialismo", G.E.G., t. 28.

s.v. “Partido Carlista Galego", G.E.G., t. 24.

C) El exilio:

Castelao, Sempre en Galiza. Buenos Aires, Eds. Galiza do Centro Galego de $1976,5 .^{\circ}$ ed.

- "As cartas de América. Documentación e fotografía", A Nosa Cultura 12. Vigo, A Nosa Terra, 1989 (introducción de J. G. BERAMENDI, «Estructura e evolución da ideoloxía política de Castelao").

Cupeiro, B., A Galicia de Alén Mar. Sada-A Coruña, Eds. do Castro, 1989. 
Díaz Pardo, 1., «Algunhas notas para os finais do exilio galego», $\boldsymbol{A}$ nosa historia 2. Os anos despois (1936-1953)...

Fernandez del Riego, F., «A cultura galega no exilio", en Díaz Pardo, I., Galicia Hoy. Sada-A Coruña, Eds. do Castro, 1987.

GónZALEZ LOPEZ, E., "La guerra civil española y la emigración gallega en América", en Galicia Eterna. Historia. Barcelona, Planeta, 1980.

Neira VILAS, X., Castelao en Cuba. Sada-A Coruña, Eds. do Castro, 1983.

Nuñez BuA, X., Vida e paixón de Castelao. Sada-A Coruña, Eds. do Castro, 1986 (reproducción de un artículo aparecido originariamente en un n. $^{\circ}$ especial de $A$ Nosa Terra editado en Buenos Aires, 25-71950).

NúÑEZ SEIXAS, X. M., "A supervivéncia do nacionalismo galego na emigración americana, 1939-1960", en TUSELL, J., ALTED, A. y MATEOS, A., La oposición al régimen de Franco. Actas Congreso Internacional. Madrid, UNED, 1990.

MARTiNez LOPEZ, R., "Literatura gallega en el exilio", en ABELLAN, J. L. (coord.), El exilio español de 1939, t. 6. Madrid, Taurus, 1978.

Palmas, R., Castelao. Prosa del exilio. Montevideo, Patronato da Cultura Galega, 1976.

- A emigración galega na Arxentina. Sada-A Coruña, Eds. do Castro, 1978.

Paz Andrade, V., Castelao na luz e na sombra. Sada-A Coruña, Eds. do Castro, 1982.

Portela Valladares, M., Dietario de dos guerras (1916-1950). Sada-A Coruña, Eds. do Castro, 1988.

SEOANE, L., Galicia emigrante. Escolma de textos da audición radial de Luis Seoane. Sada-A Coruña, Eds. do Castro,

SiXIREI, C., Alfredo Somoza. Sada-A Coruña, Eds. do Castro, 1987.

Soto, L., Castelao, a U.P.G. e outras memorias. Vigo, Eds. Xerais, 1983.

Vilanova Rodríguez, A., Los gallegos en la Argentina, 2 vols. Buenos Aires, Eds. del Centro Gallego de Buenos Aires, 1966.

Vilanova, A., "Los exiliados gallegos», en Díaz Pardo, I., Galicia Hoy. Sada-A Coruña, Eds. do Castro, 1987.

Zubillaga BarRera, C., Los gallegos en el Uruguay. Montevideo, Eds. del Patronato da Cultura Galega, 1966. 